\title{
An IMEX scheme for reaction-diffusion equations: application for a PEM fuel cell model
}

\author{
István Faragó ${ }^{1, *}$, Ferenc Izsák ${ }^{1, \dagger}$ Tamás Szabó ${ }^{3}$ and Ákos Kriston ${ }^{2, \ddagger}$
}

February 10, 2012

${ }^{1}$ Department of Applied Analysis and Computational Mathematics

${ }^{2}$ Department of Physical Chemistry

Eötvös Loránd University

H-1117, Budapest, Pázmány P. s. 1/c. Hungary

3 BCAM - Basque Center for Applied Mathematics

Alameda Mazarredo, 14, E - 48009 Bilbao

Basque Country, Spain

e-mail: faragois@cs.elte.hu, izsakf@cs.elte.hu, tszabo@bcamath.org, krisah@icegel.hu

\begin{abstract}
An implicit-explicit (IMEX) method is developed for the numerical solution of reaction-diffusion equations with pure Neumann boundary conditions. The corresponding method of lines scheme with finite differences is analyzed: explicit conditions are given for its convergence in the $\|\cdot\|_{\infty}$ norm. The results are applied to a model for determining the overpotential in a PEM fuel cell.
\end{abstract}

\section{Introduction}

The numerical solution of advection-reaction-diffusion equations is a central problem in the numerical analysis. The numerical treatment of the boundary layer effect and the possibly stiff terms lead to challenging problems. The importance of this topic lies in the applicability of the corresponding models in the natural sciences.

A possible way to build an efficient numerical solution is to apply an implicit-explicit (IMEX) method [2]. In such a procedure, the advection and diffusion terms are discretized implicitly in time and the reaction terms explicitly.

In the most simple case one can apply a $\Theta$ method for the diffusion term, which is analyzed in details in the classical paper [5].

\footnotetext{
* The first author was supported by Hungarian National Research Fund OTKA No. K67819.

${ }^{\dagger}$ The first, second and the third author was supported by the European Union and co-financed by the European Social Fund (grant agreement no. TAMOP 4.2.1./B-09/1/KMR-2010-0003).

$\ddagger$ The Financial support of the National Office of Research and Technology (OMFB-00121-00123/2008) is acknowledged by the authors.
} 
The most popular approach is the construction of corresponding Runge-Kutta methods. A family of such schemes is introduced in [1]. A corresponding rigorous convergence theory for reaction-diffusion equations was developed in [6] based on the analysis in [3] and [4]. In the above results the reaction term is assumed to have bounded derivative with respect to the unknown function. Note that IMEX methods can also be used to prove existence-uniqueness results to a class of semilinear parabolic PDE's [10].

We extend here the above results in the sense that we use a general elliptic operator with time and space dependent coefficients and a staggered grid is utilized in order to enhance the the order of consistency: Our approach guarantees a first order convergence in time and a second order in space. Note that using diagonally implicit Runge-Kutta methods can ensure only a second order convergence with respect to the time variable [12]. Also, the assumptions on the coefficients for the convergence are made explicit, which can be easily verified.

As an interesting model problem we consider a one-dimensional model for the potential distribution of a PEM fuel cell. We will have first to convert the conservation equations into a form of reaction-diffusion equations and verify that the conditions in the corresponding analysis are satisfied. The computational experiments confirm the applicability of our scheme and the theoretical results.

\section{Preliminaries}

We investigate the finite difference approximation of the reaction-diffusion equation

$$
\left\{\begin{array}{l}
\partial_{t} u(t, x)=p(t, x) \partial_{x}\left(q(t, x) \partial_{x} u(t, x)\right)+F(t, x, u(t, x)), \quad t \in(0, T), x \in I \\
u(0, x)=u_{0}(x), \quad x \in I \\
\partial_{x} u\left(t, h_{l}\right)=u_{l}(t), \partial_{x} u\left(t, h_{r}\right)=u_{r}(t), \quad t \in(0, T),
\end{array}\right.
$$

for the unknown function $u$ on the interval $I=\left(h_{l}, h_{r}\right) \subset \mathbb{R}$ over the time domain $[0, T)$, where the coefficient functions $p, q \in C^{1}([0, T] \times I)$, the reaction term $F \in C^{1}([0, T] \times I \times \mathbb{R})$ and the fluxes $u_{l}, u_{r} \in C^{1}[0, T]$ are given.

For the numerical approximation we use a staggered grid: $I$ is divided into $n$ uniform subintervals of length $h=\frac{h_{r}-h_{l}}{n}$ such that

$$
h_{j}:=h_{l}+\frac{2 j-1}{2|I|}, j=1,2, \ldots, n \quad \text { and } \quad h_{j+\frac{1}{2}}:=h_{l}+\frac{j}{|I|}, j=0,1, \ldots, n
$$

denote the midpoints and the endpoints of the subintervals, respectively as shown in the following figure:

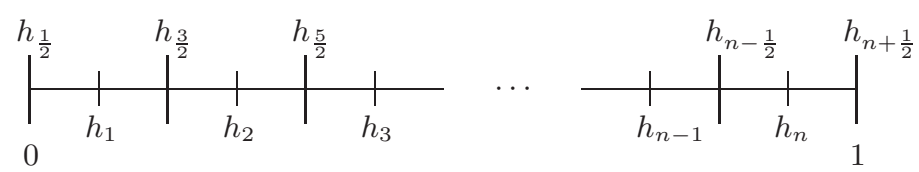

For the time discretization we use the time step $\tau=\frac{T}{N}$ and the notation $t_{k}:=\tau \cdot k$.

We denote the vector of unknowns with

$$
\boldsymbol{u}^{k}=\left(u_{1}^{k}, u_{2}^{k}, \ldots, u_{n}^{k}\right)
$$


where $u_{j}^{k} \approx u\left(t_{k}, h_{j}\right)$ and the values of the coefficient function $p_{j}^{k}=p\left(t_{k}, h_{j}\right)$ which are defined in the midpoints of the subintervals, i.e., $k=0,1, \ldots, N$ and $j=1,2, \ldots n$. Accordingly, we use the notations

$$
\boldsymbol{u}(k, \cdot)=\left(u\left(t_{k}, h_{1}\right), u\left(t_{k}, h_{2}\right), \ldots, u\left(t_{k}, h_{n}\right)\right)^{T}
$$

and

$$
\boldsymbol{F}\left(t_{k+1}, h, \boldsymbol{u}^{k}\right)=\left(F\left(t_{k+1}, h_{1}, u_{1}^{k}\right), \ldots, F\left(t_{k+1}, h_{n}, u_{n}^{k}\right)\right)^{T} .
$$

At the same time the values of the coefficient function $q_{j+\frac{1}{2}}^{k}=q\left(t_{k}, h_{j+\frac{1}{2}}\right)$ are computed at the end points of the subintervals, i.e., $k=0,1, \ldots, N$ and $j=0,1, \ldots, n$.

\section{The IMEX scheme and its convergence}

We develop here a finite difference scheme following the method of lines: the vector of unknowns at the $k+1$ th time step is determined from that in the $k$ th time step.

Using the notations in Section 2 we consider the following finite difference approximation of (1):

$$
\left\{\begin{aligned}
u_{j}^{0}=u_{0}\left(h_{j}\right), \quad j=1,2,3, \ldots, n \\
\frac{u_{j}^{k+1}-u_{j}^{k}}{\tau}=\frac{1}{h} p_{j}^{k+1}\left(q_{j+\frac{1}{2}}^{k+1} \frac{u_{j+1}^{k+1}-u_{j}^{k+1}}{h}-q_{j-\frac{1}{2}}^{k+1} \frac{u_{j}^{k+1}-u_{j-1}^{k+1}}{h}\right) \\
\quad+F\left(t_{k+1}, h_{j}, u_{j}^{k}\right), \quad k=0,1, \ldots, N-1, j=2,3, \ldots, n-1 \\
\frac{u_{1}^{k+1}-u_{1}^{k}}{\tau}=\frac{1}{h} p_{1}^{k+1}\left(q_{\frac{3}{2}}^{k+1}\left(\frac{u_{2}^{k+1}-u_{1}^{k+1}}{h}+\frac{\frac{3}{23} u_{2}^{k+1}-\frac{2}{23} u_{1}^{k+1}-\frac{1}{23} u_{3}^{k+1}}{h}-\frac{1}{23} u_{l}\left(t_{k+1}\right)\right)\right. \\
\left.\quad-q_{\frac{1}{2}}^{k+1} u_{l}\left(t_{k+1}\right)\right)+F\left(t_{k+1}, h_{1}, u_{1}^{k}\right), \quad k=0,1, \ldots, N-1 \\
\frac{u_{n}^{k+1}-u_{n}^{k}}{\tau}=\frac{1}{h} p_{n}^{k+1}\left(-q_{n-\frac{1}{2}}^{k+1}\left(\frac{u_{n}^{k+1}-u_{n-1}^{k+1}}{h}-\frac{\frac{3}{23} u_{n-1}^{k+1}-\frac{1}{23} u_{n-2}^{k+1}-\frac{2}{23} u_{n}^{k+1}}{h}-\frac{1}{23} u_{r}\left(t_{k+1}\right)\right)\right. \\
\left.\quad+q_{n+\frac{1}{2}}^{k+1} u_{r}\left(t_{k+1}\right)\right)+F\left(t_{k+1}, h_{n}, u_{n}^{k}\right), \quad k=0,1, \ldots, N-1
\end{aligned}\right.
$$

For the verification of the consistency order for the scheme (2) we will use the following

Assumption $1 \partial_{3} F: \mathbb{R}^{3} \rightarrow \mathbb{R}$ is bounded; $\partial_{3} F \leq F_{\max } \in \mathbb{R}$.

Note that a similar assumption is usual in the literature, see, e.g., [5], [6].

Lemma 3.1 The scheme (2) is consistent with the boundary value problem (1) and the corresponding order of consistency is $\mathcal{O}(\tau)+\mathcal{O}\left(h^{2}\right)$.

Proof: We obviously have that

$$
\begin{aligned}
& q_{j+\frac{1}{2}}^{k+1} \frac{u\left(t_{k+1}, h_{j+1}\right)-u\left(t_{k+1}, h_{j}\right)}{h} \\
& =q_{j+\frac{1}{2}}^{k+1}\left(\partial_{x} u\left(t_{k+1}, h_{j}\right)+\frac{h}{2} \partial_{x x} u\left(t_{k+1}, h_{j}\right)+\frac{h^{2}}{6} \partial_{x x x} u\left(t_{k+1}, h_{j}\right)+\mathcal{O}\left(h^{3}\right)\right)
\end{aligned}
$$

and in the same way

$$
\begin{aligned}
& q_{j-\frac{1}{2}}^{k+1} \frac{u\left(t_{k+1}, h_{j}\right)-u\left(t_{k+1}, h_{j-1}\right)}{h} \\
& =q_{j-\frac{1}{2}}^{k+1}\left(\partial_{x} u\left(t_{k+1}, h_{j}\right)-\frac{h}{2} \partial_{x x} u\left(t_{k+1}, h_{j}\right)+\frac{h^{2}}{6} \partial_{x x x} u\left(t_{k+1}, h_{j}\right)+\mathcal{O}\left(h^{3}\right)\right) .
\end{aligned}
$$


The difference of (3) and (4) gives that

$$
\begin{aligned}
& \frac{1}{h}\left(q_{j+\frac{1}{2}}^{k+1} \frac{u\left(t_{k+1}, h_{j+1}\right)-u\left(t_{k+1}, h_{j}\right)}{h}-q_{j-\frac{1}{2}}^{k+1} \frac{u\left(t_{k+1}, h_{j}\right)-u\left(t_{k+1}, h_{j-1}\right)}{h}\right) \\
& =\frac{q_{j+\frac{1}{2}}^{k+1}-q_{j-\frac{1}{2}}^{k+1}}{h} \partial_{x} u\left(t_{k}, h_{j}\right)+\frac{q_{j+\frac{1}{2}}^{k+1}+q_{j-\frac{1}{2}}^{k+1}}{2} \partial_{x x} u\left(t_{k}, h_{j}\right) \\
& +\frac{h}{6}\left(q_{j+\frac{1}{2}}^{k+1}-q_{j-\frac{1}{2}}^{k+1}\right) \partial_{x x x} u\left(t_{k}, h_{j}\right)+\mathcal{O}\left(h^{2}\right) \\
& =\left(\partial_{x} q\left(t_{k+1}, h_{j}\right)+\mathcal{O}\left(h^{2}\right)\right) \partial_{x} u\left(t_{k}, h_{j}\right)+\left(q\left(t_{k+1}, h_{j}\right)+\mathcal{O}\left(h^{2}\right)\right) \partial_{x x} u\left(t_{k}, h_{j}\right) \\
& +\frac{h}{6} \mathcal{O}(h) \partial_{x x x} u\left(t_{k}, h_{j}\right)+\mathcal{O}\left(h^{2}\right) \\
& =\partial_{x}\left(q\left(t_{k+1}, h_{j}\right) \partial_{x} u\left(t_{k}, h_{j}\right)\right)+\mathcal{O}\left(h^{2}\right)
\end{aligned}
$$

for $j=2,3, \ldots, n-1$. Therefore, inserting the analytic solution of (1) at $\left(t_{k+1}, h_{j}\right)$ into $(2)$ results in the approximation

$$
\begin{aligned}
& \frac{u\left(t_{k+1}, h_{j}\right)-u\left(t_{k}, h_{j}\right)}{\tau}+\mathcal{O}(\tau)=\partial_{t} u\left(t_{k+1}, h_{j}\right)=p_{j}^{k+1} \partial_{x}\left(q_{j}^{k+1} \partial_{x} u\left(t_{k+1}, x_{j}\right)\right)+F\left(t_{k+1}, x_{j}, u\left(t_{k+1}, x_{j}\right)\right) \\
& =\frac{1}{h} p_{j}^{k+1}\left(q_{j+\frac{1}{2}}^{k+1} \frac{u\left(t_{k+1}, h_{j+1}\right)-u\left(t_{k+1}, h_{j}\right)}{h}-q_{j-\frac{1}{2}}^{k+1} \frac{u\left(t_{k+1}, h_{j}\right)-u\left(t_{k+1}, h_{j-1}\right)}{h}\right)+\mathcal{O}\left(h^{2}\right) \\
& +F\left(t_{k+1}, h_{j}, u\left(t_{k+1}, h_{j}\right)\right), \quad k=0,1, \ldots, N-1, j=2,3, \ldots, n-1 .
\end{aligned}
$$

To approximate the last term we note that $u\left(t_{k+1}, h_{j}\right)-u\left(t_{k}, h_{j}\right)=\mathcal{O}(\tau)$ and therefore, using Assumption 1 we easily get

$$
\begin{aligned}
F\left(t_{k+1}, h_{j}, u\left(t_{k+1}, h_{j}\right)\right) & =F\left(t_{k+1}, h_{j}, u\left(t_{k}, h_{j}\right)\right)+\partial_{3} F\left(t_{k+1}, h_{j}, u\left(t_{k}, h_{j}\right)\right) \cdot \mathcal{O}(\tau)+\mathcal{O}\left(\tau^{2}\right) \\
& =F\left(t_{k+1}, h_{j}, u\left(t_{k}, h_{j}\right)\right)+\mathcal{O}(\tau) .
\end{aligned}
$$

Inserting this into (6) we obtain

$$
\begin{aligned}
& \frac{u\left(t_{k+1}, h_{j}\right)-u\left(t_{k}, h_{j}\right)}{\tau}=\frac{1}{h} p_{j}^{k+1}\left(q_{j+\frac{1}{2}}^{k+1} \frac{u\left(t_{k+1}, h_{j+1}\right)-u\left(t_{k+1}, h_{j}\right)}{h}-q_{j-\frac{1}{2}}^{k+1} \frac{u\left(t_{k+1}, h_{j}\right)-u\left(t_{k+1}, h_{j-1}\right)}{h}\right) \\
& +F\left(t_{k+1}, h_{j}, u\left(t_{k}, h_{j}\right)\right)+\mathcal{O}\left(h^{2}\right)+\mathcal{O}(\tau), \quad k=0,1, \ldots, N-1, j=2,3, \ldots, n-1 .
\end{aligned}
$$

We also have

$$
\begin{aligned}
& q_{\frac{1}{2}}^{k+1}\left(u_{l}\left(t_{k+1}\right)\right)=q_{\frac{1}{2}}^{k+1}\left(\partial_{x} u\left(t_{k+1}, h_{\frac{1}{2}}\right)\right) \\
& =q_{\frac{1}{2}}^{k+1}\left(\partial_{x} u\left(t_{k+1}, h_{1}\right)-\frac{h}{2} \partial_{x x} u\left(t_{k+1}, h_{1}\right)+\frac{h^{2}}{8} \partial_{x x x} u\left(t_{k+1}, h_{1}\right)+\mathcal{O}\left(h^{3}\right)\right) .
\end{aligned}
$$

and the following equality:

$$
\begin{aligned}
& \frac{1}{h}\left(\frac{3}{23} u\left(t_{k+1}, h_{2}\right)-\frac{2}{23} u\left(t_{k+1}, h_{1}\right)-\frac{1}{23} u\left(t_{k+1}, h_{3}\right)\right)-\frac{1}{23} \partial_{x} u\left(t_{k+1}, h_{\frac{1}{2}}\right) \\
& =-\frac{h^{2}}{24}\left(\partial_{x x x} u\left(t_{k+1}, h_{1}\right)+\mathcal{O}(h)\right)
\end{aligned}
$$


which can be verified with a technical but straightforward computation. Using (3) with $j=1,(8),(9)$ and following the principle of the derivation in (5) we obtain

$$
\begin{aligned}
& q_{\frac{3}{2}}^{k+1}\left(\frac{u\left(t_{k+1}, h_{2}\right)-u\left(t_{k+1}, h_{1}\right)}{h}+\frac{\frac{3}{23} u_{2}-\frac{2}{23} u_{1}-\frac{1}{23} u_{3}}{h}-\frac{1}{23} u_{l}\left(t_{k+1}\right)\right) \\
& -q_{\frac{1}{2}}^{k+1} \partial_{x} u\left(t_{k+1}, h_{l}\right) \\
& =q_{\frac{3}{2}}^{k+1}\left(\partial_{x} u\left(t_{k+1}, h_{1}\right)-\frac{h}{2} \partial_{x x} u\left(t_{k+1}, h_{1}\right)+\frac{h^{2}}{6} \partial_{x x x} u\left(t_{k+1}, h_{1}\right)+\mathcal{O}\left(h^{3}\right)\right. \\
& \left.-\frac{h^{2}}{24}\left(\partial_{x x x} u\left(t_{k+1}, h_{1}\right)+\mathcal{O}(h)\right)\right) \\
& -q_{\frac{1}{2}}^{k+1}\left(\partial_{x} u\left(t_{k+1}, h_{1}\right)+\frac{h}{2} \partial_{x x} u\left(t_{k+1}, h_{1}\right)+\frac{h^{2}}{8} \partial_{x x x} u\left(t_{k+1}, h_{1}\right)+\mathcal{O}\left(h^{3}\right)\right) \\
& =q_{\frac{3}{2}}^{k+1}\left(\partial_{x} u\left(t_{k+1}, h_{1}\right)-\frac{h}{2} \partial_{x x} u\left(t_{k+1}, h_{1}\right)+\frac{h^{2}}{8} \partial_{x x x} u\left(t_{k+1}, h_{1}\right)+\mathcal{O}\left(h^{3}\right)\right) \\
& -q_{\frac{1}{2}}^{k+1}\left(\partial_{x} u\left(t_{k+1}, h_{1}\right)+\frac{h}{2} \partial_{x x} u\left(t_{k+1}, h_{1}\right)+\frac{h^{2}}{8} \partial_{x x x} u\left(t_{k+1}, h_{1}\right)+\mathcal{O}\left(h^{3}\right)\right) \\
& =h \cdot \partial_{x}\left(q\left(t_{k+1}, h_{1}\right) \partial_{x} u\left(t_{k}, h_{1}\right)\right)+\mathcal{O}\left(h^{3}\right), \quad k=0,1, \ldots, N-1 .
\end{aligned}
$$

Using that

$$
\begin{aligned}
& \frac{1}{h}\left(\frac{3}{23} u\left(t_{k+1}, h_{n-1}\right)-\frac{2}{23} u\left(t_{k+1}, h_{n}\right)-\frac{1}{23} u\left(t_{k+1}, h_{n-2}\right)\right)+\frac{1}{23} \partial_{x} u\left(t_{k+1}, h_{n+\frac{1}{2}}\right) \\
& =\frac{h^{2}}{24}\left(\partial_{x x x} u\left(t_{k+1}, h_{1}\right)+\mathcal{O}(h)\right)
\end{aligned}
$$

a similar derivation gives that

$$
\begin{aligned}
& -q_{n-\frac{1}{2}}^{k+1}\left(\frac{u_{n}^{k+1}-u_{n-1}^{k+1}}{h}-\frac{\frac{3}{23} u_{n-1}^{k+1}-\frac{1}{23} u_{n-2}^{k+1}-\frac{2}{23} u_{n}^{k+1}}{h}-\frac{1}{23} u_{r}\left(t_{k+1}\right)\right)+q_{n+\frac{1}{2}}^{k+1} u_{r}\left(t_{k+1}\right) \\
& =h \cdot \partial_{x}\left(q\left(t_{k+1}, h_{n}\right) \partial_{x} u\left(t_{k}, h_{n}\right)\right)+\mathcal{O}\left(h^{3}\right), \quad k=0,1, \ldots, N-1 .
\end{aligned}
$$

Consequently, (7), (10) and (11) imply that the finite difference approximation in (2) is consistent with the initial boundary value problem (1) and the order of the consistency is $\mathcal{O}(\tau)+\mathcal{O}\left(h^{2}\right)$ as stated in the lemma.

To rewrite (2) into a more accessible form we introduce the notations for $j=1,2, \ldots, n$ :

$$
r p_{j}^{k} q_{j-\frac{1}{2}}^{k}=c_{j}^{k} \quad \text { and } \quad r p_{j}^{k} q_{j+\frac{1}{2}}^{k}=d_{j}^{k} \quad \text { with } r=\frac{\tau}{h^{2}} .
$$


With these we define the matrix

$$
A_{k, h}=\left(\begin{array}{cccccc}
1+\frac{25}{23} d_{1}^{k} & -\frac{26}{23} d_{1}^{k} & \frac{1}{23} d_{1}^{k} & 0 & \ldots & 0 \\
-c_{2}^{k} & 1+c_{2}^{k}+d_{2}^{k} & -d_{2}^{k} & 0 & \ldots & 0 \\
0 & -c_{3}^{k} & 1+c_{3}^{k}+d_{3}^{k} & -d_{3}^{k} & \ldots & 0 \\
\vdots & \vdots & \vdots & \vdots & \ddots & \vdots \\
0 & \cdots & 0 & -c_{n-1}^{k} & 1+c_{n-1}^{k}+d_{n-1}^{k} & -d_{n-1}^{k} \\
0 & \cdots & 0 & \frac{1}{23} c_{n}^{k} & -\frac{26}{23} c_{n}^{k} & 1+\frac{25}{23} c_{n}^{k}
\end{array}\right)
$$

and the vector

$$
\boldsymbol{v}^{k}=\left(\frac{\tau}{h} p_{1}^{k}\left(q_{\frac{3}{2}}^{k} \cdot \frac{1}{23} \cdot u_{l}\left(t_{k}\right)+q_{\frac{1}{2}}^{k} u_{l}\left(t_{k}\right)\right), 0, \ldots, 0,-\frac{\tau}{h} p_{n}^{k}\left(q_{n-\frac{1}{2}}^{k} \cdot \frac{1}{23} \cdot u_{r}\left(t_{k}\right)+q_{n+\frac{1}{2}}^{k} u_{r}\left(t_{k}\right)\right)^{T} .\right.
$$

The time stepping in (2) then can be given as

$$
\boldsymbol{u}^{k}=A_{k+1, h} \boldsymbol{u}^{k+1}-\tau \boldsymbol{F}\left(t, h, \boldsymbol{u}^{k}\right)+\boldsymbol{v}^{k+1} .
$$

For the forthcoming analysis we use two further assumptions:

Assumption 2 The coefficient functions $p$ and $q$ are nonnegative.

Assumption 3 For all $k=1,2, \ldots, N$ the following inequalities hold true:

$$
\begin{array}{r}
s_{1}^{k}=\frac{25}{23} d_{1}^{k}-\frac{1}{23} \frac{d_{1}^{k}}{d_{2}^{k}}-\frac{1}{23} \frac{d_{1}^{k} c_{2}^{k}}{d_{2}^{k}}>0 \\
s_{2}^{k}=\frac{25}{23} c_{n}^{k}-\frac{1}{23} \frac{c_{n}^{k}}{c_{n-1}^{k}}-\frac{1}{23} \frac{c_{n}^{k} d_{n-1}^{k}}{c_{n-1}^{k}}>0
\end{array}
$$

Remark: The inequalities in assumption 3 are equivalent with

$$
\begin{array}{r}
25 d_{2}^{k}>1+c_{2}^{k} \Leftrightarrow r p_{2}\left(25 q_{\frac{5}{2}}-q_{\frac{3}{2}}\right)>1 \\
25 c_{n-1}^{k}>1+d_{n-1}^{k} \Leftrightarrow r p_{n-1}\left(25 q_{n-\frac{1}{2}}-q_{n+\frac{1}{2}}\right)>1
\end{array}
$$

For the convergence proof we need to rewrite (2) in a more compact form. The following property of $A_{k, h}$ is of central importance.

Lemma 3.2 For all $h>0$ and $k=0,1, \ldots, N$ we have $\left\|A_{k, h}^{-1}\right\|_{\infty}=1$.

Proof: One can easily verify that

$$
A_{k, h}=\left(\begin{array}{ccccc}
1 & -\lambda_{1}^{k} & 0 & \ldots & 0 \\
0 & 1 & 0 & \ldots & 0 \\
\vdots & \vdots & \vdots & \ddots & \vdots \\
0 & \ldots & 0 & 1 & 0 \\
0 & \ldots & 0 & -\lambda_{2}^{k} & 1
\end{array}\right)\left(\begin{array}{ccccc}
1+s_{1}+\lambda_{1}^{k} & -s_{1} & 0 & \ldots & 0 \\
-c_{2}^{k} & 1+c_{2}^{k}+d_{2}^{k} & -d_{2}^{k} & \ldots & 0 \\
\vdots & \vdots & \vdots & \ddots & \vdots \\
0 & \ldots & -c_{n-1}^{k} & 1+c_{n-1}^{k}+d_{n-1}^{k} & -d_{n-1}^{k} \\
0 & \ldots & 0 & -s_{2}^{k} & 1+s_{2}^{k}+\lambda_{2}^{k}
\end{array}\right),
$$


where $\lambda_{1}^{k}=\frac{1}{23} \frac{d_{1}^{k}}{d_{2}^{k}}$ and $\lambda_{2}^{k}=\frac{1}{23} \frac{c_{n}^{k}}{c_{n-1}^{k}}$. Using Assumption 2 and Assumption 3 and the vector $\mathbb{1}=$ $(1,1, \ldots, 1)^{T}$, we obtain that the second matrix in the above product is an $M$ matrix. Therefore, the decomposition

$$
A_{k, h}^{-1}=\left(\begin{array}{ccccc}
1+s_{1}+\lambda_{1}^{k} & -s_{1} & 0 & \ldots & 0 \\
-c_{2}^{k} & 1+c_{2}^{k}+d_{2}^{k} & -d_{2}^{k} & \ldots & 0 \\
\vdots & \vdots & \vdots & \ddots & \vdots \\
0 & \ldots & -c_{n-1}^{k} & 1+c_{n-1}^{k}+d_{n-1}^{k} & -d_{n-1}^{k} \\
0 & \ldots & 0 & -s_{2}^{k} & 1+s_{2}^{k}+\lambda_{2}^{k}
\end{array}\right)^{-1}\left(\begin{array}{ccccc}
1 & \lambda_{1}^{k} & 0 & \ldots & 0 \\
0 & 1 & 0 & \ldots & 0 \\
\vdots & \vdots & \vdots & \ddots & \vdots \\
0 & \ldots & 0 & 1 & 0 \\
0 & \ldots & 0 & \lambda_{2}^{k} & 1
\end{array}\right)
$$

shows that $A_{k, h}^{-1}$ has positive entries. Since $A_{k, h} \mathbb{1}=\mathbb{1}$ we also have $A_{k, h}^{-1} \mathbb{1}=\mathbb{1}$ which implies the statement of the lemma.

Theorem 3.1 The finite difference method given by (2) converges to the solution of (1) and

$$
\max _{j \in\{1,2, \ldots, n\}}\left\|u_{j}^{N}-u\left(T, h_{j}\right)\right\|=\mathcal{O}(\tau)+\mathcal{O}\left(h^{2}\right) .
$$

Proof: The error of the solution in the consecutive time steps is defined as

$$
\left(e_{1}^{k}, e_{2}^{k}, \ldots, e_{n}^{k}\right)=e^{k}=u(k, \cdot)-u^{k} .
$$

The consistency of the scheme implies that

$$
\boldsymbol{u}(k, \cdot)=A_{k+1, h} \boldsymbol{u}(k+1, \cdot)-\tau \boldsymbol{F}(t, h, \boldsymbol{u}(k, \cdot))+\boldsymbol{v}_{k+1}-\mathcal{R}^{k},
$$

where

$$
\left\|\mathcal{R}^{k}\right\|_{\infty}=\tau\left(\mathcal{O}(\tau)+\mathcal{O}\left(h^{2}\right)\right)
$$

This together with (12) gives that

$$
\boldsymbol{e}^{k}=A_{k+1, h} \boldsymbol{e}^{k+1}-\tau\left(\boldsymbol{F}\left(t, h, \boldsymbol{u}^{k}\right)-\boldsymbol{F}(t, h, \boldsymbol{u}(k, \cdot))\right)+\mathcal{R}^{k}
$$

or in an equivalent form

$$
\boldsymbol{u}^{k}-\boldsymbol{u}(k, \cdot)=\boldsymbol{e}^{k+1}=A_{k+1, h}^{-1} \boldsymbol{e}^{k}+\tau A_{k+1, h}^{-1}\left(\boldsymbol{F}\left(t, h, \boldsymbol{u}^{k}\right)-\boldsymbol{F}(t, h, \boldsymbol{u}(k, \cdot))\right)+\mathcal{R}^{k+1} .
$$

Therefore, using the result in Lemma 3.2, the Lagrange inequality and Assumption 1 we obtain

$$
\left\|\boldsymbol{e}^{k+1}\right\|_{\infty} \leq\left\|\boldsymbol{e}^{k}\right\|_{\infty}+\tau F_{\max }\left\|\boldsymbol{e}^{k}\right\|_{\infty}+\left\|\mathcal{R}^{k+1}\right\|_{\infty}
$$

for all $k=1,2, \ldots, N$. The consecutive application of (16) gives that

$$
\begin{aligned}
\left\|\boldsymbol{e}^{N}\right\|_{\infty} & \leq\left(1+\tau F_{\max }\right)^{N-1}\left\|\mathcal{R}^{1}\right\|_{\infty}+\left(1+\tau F_{\max }\right)^{N-2}\left\|\mathcal{R}^{2}\right\|_{\infty}+\cdots+\left\|\mathcal{R}^{N}\right\|_{\infty} \\
& \leq N\left(1+\tau F_{\max }\right)^{N} \max _{j \in\{1,2, \ldots, N\}}\left\|\mathcal{R}^{j}\right\|_{\infty} \leq T e^{T \cdot F_{\max }} \frac{\max _{j \in\{1,2, \ldots, N\}}\left\|\mathcal{R}^{j}\right\|_{\infty}}{\tau}
\end{aligned}
$$

such that according to (15) we obtain the estimate in the theorem. 


\section{Application}

In this section we investigate a real-life application: we compute numerically the overpotential in PEM fuel cell. Fuel cells "burn" hydrogen fuel $\left(\mathrm{H}_{2}\right)$ and oxygen $\left(\mathrm{O}_{2}\right)$ to water, producing electrical energy at a high efficiency without air pollution. Their operation can be reversible: they can also convert electrical energy into chemical energy. These properties make them highly attractive.

The electro-chemical reactions take place at the anode and at the cathode on the boundary of two phases (solid and solution phase), while the charge neutrality is macroscopically preserved.

Complex models [13] are needed to solve different phenomenological equations such as the Nernst-Planck equation for multiple mass transport, the Stefan-Maxwell equation for heat transfer, Ohm's law for ionic migration and electron conductivity, and the equations of electrochemical kinetics. These models are usually solved by using only a single numerical treatment, e.g., Runge-Kutta, Newton or Crank-Nicholson methods.

Subramanian et al. [11] developed a method to reduce the number of the governing equations of Liion battery simulation by using different mathematical techniques. The original problem with a proper discretization has 4800 equations which can be reduced to 49, and finally the simulation time of the discharge curve can be cut to $85 \mathrm{~ms}$. However, in this model the double-layer capacitance was not included.

We focus here only on the evolution of the overpotential and we take into consideration both the inhomogeneity of the conducting media and the presence of the different phases in the cell. We perform the computations with realistic parameters.

\subsection{Physical laws: homogeneous and heterogeneous models}

In practice a consumer (an electric device) is inserted into an electrical circuit, which is feeded by the fuel cell. We assume that the current in the outer circuit is known and we can control it. The aim of the following investigation is to calculate the corresponding voltage, which is called the cell potential. This gives also the electric energy provided by the fuel cell, which is very important in the course of evaluating the performance of a fuel cell.

According to Kirchoff's law, the cell potential $E_{\text {cell }}$ can be calculated by the following equation, see also $[8]:$

$$
E_{\mathrm{cell}}(t)=E_{\mathrm{OC}}(t)-\eta^{a}(t)-\frac{W_{\mathrm{mem}}}{\kappa_{\mathrm{mem}}} I(t)-V^{*}(t),
$$

where $t \in(0, T)$ denotes time. Here $E_{\mathrm{OC}} \approx 1.23 \mathrm{~V}$ denotes the open circuit potential, which is present between the anode and cathode without the presence of any consumer.

Considering the simplest form of Ohm's law, the term $\frac{W_{\text {mem }}}{\kappa_{\text {mem }}} I(t)$ means the potential loss at the membrane, the thickness and conductivity of which are denoted by $W_{\text {mem }}$ and $\kappa_{\text {mem }}$, respectively. The calculation of the last quantity on the right-hand side $\left(V^{*}\right)$, which refers to the potential loss at the cathode, needs a detailed analysis. The interval $(0, L)$ refers to the thickness of the cathode, where two phases are distinguished:

- The solution phase, where the hydrogen ions are conducted according to the rate $\kappa_{\text {eff }}$. The potential and the current density in this phase are denoted by $\phi_{2}$ and $i_{2}$, respectively.

- In the solid phase of the cathode electrons are conducted according to the rate $\sigma_{\text {eff. }}$ The potential and the current density here are denoted by $\phi_{1}$ and $i_{1}$, respectively. 
All of these quantities could be allowed to depend on time and space corresponding to the given assumptions and the structure of the fuel cell and the time evolution of the process.

Using the defined quantities, $V^{*}$ in $(17)$ can be given as

$$
V^{*}(t)=\phi_{1}(t, L)-\phi_{2}(t, 0), \quad t \in(0, T)
$$

The quantity we investigate in the governing equations is the overpotential

$$
\eta(t, x)=\phi_{1}(t, x)-\phi_{2}(t, x) \geq 0, \quad x \in(0, L), t \in(0, T)
$$

In the calculation of the potentials, we choose the reference level to be at the left end of the solution phase, i.e., we define $\phi_{2}(t, 0)=0$. This is in a good accordance with the uniqueness of the solutions in the corresponding equations. As we will see, the governing equations depend only on the spatial derivatives of the potentials, such that the above assumption is necessary to determine both $\phi_{2}(t, x)$ and $\eta(t, x)$. Then an immediate consequence of (18) and (19) is that

$$
V^{*}(t)=\phi_{1}(t, L)=\eta(t, L)+\phi_{2}(t, L) .
$$

Applying Ohm's law for both phases we obtain

$$
\begin{aligned}
& i_{1}(t, x)=-\sigma_{\mathrm{eff}}(x) \partial_{x} \phi_{1}(t, x), \\
& i_{2}(t, x)=-\kappa_{\mathrm{eff}}(x) \partial_{x} \phi_{2}(t, x),
\end{aligned}
$$

and the principle of the electroneutrality gives

$$
-\partial_{x} i_{1}(t, x)=\partial_{x} i_{2}(t, x) .
$$

The conservation law for the currents (see [9]) results in the formula

$$
\partial_{x}\left(\kappa_{\mathrm{eff}}(x) \partial_{x} \phi_{2}(t, x)\right)=-a(x) C_{\mathrm{dl}}(x) \partial_{t} \eta(t, x)-a(x) i_{0}(x) g\left(\alpha \frac{F}{R T} \eta(t, x)\right) .
$$

Here, the function $C_{\mathrm{dl}}(x)$ gives the double-layer capacitance at the cathode side, and the last term yields the faradic current with $i_{0}(x)$, the exchange current density at the cathode. For the notations of the material coefficients we refer to the Appendix. The function $g: \mathbb{R} \rightarrow \mathbb{R}$ refers to the kinetics of the oxygen reduction reaction here. This should be an increasing function with $g(0)=0$.

Remark 4.1 Among the several approaches for the sake of simplicity we apply linear kinetics and, accordingly, we use

$$
g_{L}(u)=c(x) u
$$

where $c(x)$ is a given bounded non-negative function. Other possible choices are the following, which are going to be used in the course of the analysis and the numerical experiments [7].

- Butler-Volmer kinetics:

$$
g_{B V}(u)=c(x)(\exp (u)-\exp (-u))
$$


- diffusion kinetics:

$$
g_{D}(u)=j_{D}(x)\left(\frac{c(x) \exp (u)}{c(x) \exp (u)+j_{D}(x)}-\frac{c(x) \exp (-u)}{c(x) \exp (-u)+j_{D}(x)}\right),
$$

where $j_{D}(x)$ is the limiting current, which in this equation is acting as a diffusion coefficient. This choice provides the most accurate model of the cathode reaction.

In what follows the notation $g(u)$ stands for any of the above functions $\left(g_{L}, g_{B V}, g_{D}\right)$.

At the left end of the cathode only the protons can exit to the membrane and similarly, at the right end (at the current collector), only the electrons can leave the cathode. Therefore $\partial_{x} \phi_{1}(t, 0)=0$ and $\partial_{x} \phi_{2}(t, L)=0$ such that using (19) we have the following boundary conditions

$$
\begin{aligned}
& \partial_{x} \eta(t, 0)=-\partial_{x} \phi_{2}(t, 0)=-\frac{1}{\kappa_{\text {eff }}(0)} I(t), t \in\left(0, t_{\text {max }}\right), \\
& \partial_{x} \eta(t, L)=\partial_{x} \phi_{1}(t, L)=\frac{1}{\sigma_{\text {eff }}(L)} I(t), t \in\left(0, t_{\text {max }}\right) .
\end{aligned}
$$

Although we have listed all physical principles and the governing equations here, the corresponding equations are not yet ready for the solution, since (23) contains also the unknown term $\phi_{2}(t, x)$.

\subsection{Governing equations in the heterogeneous case}

In this section we will obtain an explicit equation for the overpotential $\eta(t, x)$ by eliminating the term $\phi_{2}(t, x)$ in (23) without assuming constant material and kinetic coefficients. This generalizes the result in

Lemma 4.1 The physical laws in (21), (22), (23) and (27) can be rewritten into a single reaction diffusion equation of type (1) for the unknown function $\eta$.

Proof: For simplicity, in the derivation we denote the variables $t$ and $x$ only for the unknown functions $\phi_{2}$ and $\eta$, whenever also the coefficient functions $a, i_{0}, C_{\mathrm{dl}}, \kappa_{\mathrm{eff}}$ and $\sigma_{\mathrm{eff}}$ depend on $(t, x)$ and $I$ depends on $t$ with $t \in\left(0, t_{\max }\right)$ and $x \in(0, L)$.

Using (21) and taking the derivative of (22) we obtain that

$$
\partial_{x}\left(\sigma_{\mathrm{eff}} \partial_{x} \phi_{1}\right)=-\partial_{x} i_{1}=\partial_{x} i_{2}=-\partial_{x}\left(\kappa_{\mathrm{eff}} \partial_{x} \phi_{2}\right),
$$

which, together with the definition (19) of $\eta(t, x)$ gives

$$
\begin{aligned}
& \partial_{x}\left(\sigma_{\text {eff }} \partial_{x} \phi_{2}+\kappa_{\text {eff }} \partial_{x} \phi_{2}\right) \\
& =\partial_{x}\left(\sigma_{\text {eff }} \partial_{x} \phi_{2}\right)-\partial_{x}\left(\sigma_{\text {eff }} \partial_{x} \phi_{1}\right)=-\partial_{x}\left(\sigma_{\text {eff }} \partial_{x} \eta\right) .
\end{aligned}
$$

Since the two derivatives in (29) are equal, we obtain

$$
\begin{aligned}
& \left(\kappa_{\text {eff }}+\sigma_{\text {eff }}\right) \partial_{x} \phi_{2}(t, x) \\
& =-\sigma_{\text {eff }} \partial_{x} \eta(t, x)+\left(\kappa_{\text {eff }}+\sigma_{\text {eff }}\right) \partial_{x} \phi_{2}(t, 0)+\sigma_{\text {eff }} \partial_{x} \eta(t, 0) \\
& =-\sigma_{\text {eff }} \partial_{x} \eta(t, x)+\kappa_{\text {eff }} \partial_{x} \phi_{2}(t, 0)=-\sigma_{\text {eff }} \partial_{x} \eta(t, x),
\end{aligned}
$$


where in the second line the boundary conditions (27) have been used twice. Using (29) and (30), we rewrite the left-hand side of equation (23) as

$$
\begin{aligned}
\partial_{x}\left(\kappa_{\mathrm{eff}} \partial_{x} \phi_{2}\right) & =\partial_{x}\left(\frac{\kappa_{\mathrm{eff}}}{\kappa_{\mathrm{eff}}+\sigma_{\mathrm{eff}}} \kappa_{\mathrm{eff}} \partial_{x} \phi_{2}+\frac{\kappa_{\mathrm{eff}}}{\kappa_{\mathrm{eff}}+\sigma_{\mathrm{eff}}} \sigma_{\mathrm{eff}} \partial_{x} \phi_{2}\right) \\
& =\partial_{x}\left(\frac{\kappa_{\mathrm{eff}}}{\kappa_{\mathrm{eff}}+\sigma_{\mathrm{eff}}}\right)\left(\kappa_{\mathrm{eff}} \partial_{x} \phi_{2}+\sigma_{\mathrm{eff}} \partial_{x} \phi_{2}\right) \\
& +\frac{\kappa_{\mathrm{eff}}}{\kappa_{\mathrm{eff}}+\sigma_{\mathrm{eff}}} \partial_{x}\left(\kappa_{\mathrm{eff}} \partial_{x} \phi_{2}+\sigma_{\mathrm{eff}} \partial_{x} \phi_{2}\right) \\
& =-\partial_{x}\left(\frac{\kappa_{\mathrm{eff}}}{\kappa_{\mathrm{eff}}+\sigma_{\mathrm{eff}}}\right)\left(\sigma_{\mathrm{eff}} \partial_{x} \eta-I\right)-\frac{\kappa_{\mathrm{eff}}}{\kappa_{\mathrm{eff}}+\sigma_{\mathrm{eff}}} \partial_{x}\left(\sigma_{\mathrm{eff}} \partial_{x} \eta\right) .
\end{aligned}
$$

Substituting (31) into the left-hand side of (23), results in the explicit equation

$$
\begin{aligned}
a C_{\mathrm{dl}} \partial_{t} \eta(t, x) & =\partial_{x}\left(\frac{\kappa_{\mathrm{eff}}}{\kappa_{\mathrm{eff}}+\sigma_{\mathrm{eff}}}\right)\left(-I(t)+\sigma_{\mathrm{eff}} \partial_{x} \eta(t, x)\right) \\
& +\frac{\kappa_{\mathrm{eff}}}{\kappa_{\mathrm{eff}}+\sigma_{\mathrm{eff}}} \partial_{x}\left(\sigma_{\mathrm{eff}} \partial_{x} \eta(t, x)\right)-a i_{0} g\left(\alpha \frac{F}{R T} \eta(t, x)\right) \\
& =\partial_{x}\left[\frac{\kappa_{\mathrm{eff}} \sigma_{\mathrm{eff}}}{\kappa_{\mathrm{eff}}+\sigma_{\mathrm{eff}}} \partial_{x} \eta(t, x)\right]-\partial_{x}\left(\frac{\kappa_{\mathrm{eff}}}{\kappa_{\mathrm{eff}}+\sigma_{\mathrm{eff}}}\right) I(t) \\
& -a i_{0} g\left(\alpha \frac{F}{R T} \eta(t, x)\right)
\end{aligned}
$$

for the unknown function $\eta$. For the corresponding initial-boundary value problem we use the initial value

$$
\eta(0, x)=0, x \in(0, L),
$$

and (32) is equipped with the Neumann type boundary conditions in (27).

Remark: Based on (30), we can express $\phi_{2}(t, x)$ as

$$
\partial_{x} \phi_{2}(t, x)=\frac{1}{\kappa_{\mathrm{eff}}(x)+\sigma_{\mathrm{eff}}(x)}\left(I(t)-\sigma_{\mathrm{eff}}(x) \partial_{x} \eta(t, x)\right),
$$

and consequently, by the assumption $\phi_{2}(t, 0)=0$ (see the explanation after (19)) we have

$$
\phi_{2}(t, x)=\int_{0}^{x}\left(-\frac{\sigma_{\mathrm{eff}}(t, s)}{\kappa_{\mathrm{eff}}(t, s)+\sigma_{\mathrm{eff}}(t, s)} \partial_{s} \eta(t, s)+\frac{1}{\kappa_{\mathrm{eff}}(t, s)+\sigma_{\mathrm{eff}}(t, s)} I(t)\right) \mathrm{d} s .
$$

Therefore, according to (20) we can give the potential loss $V^{*}$ at the anode as

$$
\begin{aligned}
& V^{*}(t)=\eta(t, L)+\phi_{2}(t, L) \\
& =\eta(t, L)+\int_{0}^{L}-\frac{\sigma_{\mathrm{eff}}(t, s)}{\kappa_{\mathrm{eff}}(t, s)+\sigma_{\mathrm{eff}}(t, s)} \partial_{s} \eta(t, s)+\frac{1}{\kappa_{\mathrm{eff}}(t, s)+\sigma_{\mathrm{eff}}(t, s)} I(t) \mathrm{d} s .
\end{aligned}
$$

This completes the computation of the right-hand side of (17), and the desired quantity $E_{\text {cell }}(t)$ can be given. 
Remark: According to Lemma 4.1 we have that

$$
p=\frac{1}{a C_{\mathrm{dl}}}, \quad q=\frac{\kappa_{\mathrm{eff}} \sigma_{\mathrm{eff}}}{\kappa_{\mathrm{eff}}+\sigma_{\mathrm{eff}}} \quad \text { and } \quad F(t, x, \eta(t, x))=-\frac{i_{0}}{C_{\mathrm{dl}}} g\left(\alpha \frac{F}{R T} \eta(t, x)\right)-\frac{1}{a C_{\mathrm{dl}}} \partial_{x}\left(\frac{\kappa_{\mathrm{eff}}}{\kappa_{\mathrm{eff}}+\sigma_{\mathrm{eff}}}\right) I(t)
$$

\subsection{Model problem}

For testing the method in the article, we investigate here a model problem. Based on real measurements we have $\kappa_{\text {eff }} \approx 0.002$ and $\sigma_{\text {eff }} \approx 1.8$ and accordingly, we define

$$
\kappa_{\mathrm{eff}}(t, x)=0.002-0.001 x \quad \text { and } \quad \sigma_{\mathrm{eff}}(t, x)=1.8+0.001 x .
$$

Consequently,

$$
\kappa_{\mathrm{eff}}+\sigma_{\mathrm{eff}}=1.801 \text { and } \frac{\kappa_{\mathrm{eff}}}{\sigma_{\mathrm{eff}}+\kappa_{\mathrm{eff}}}(t, x)=\frac{2-x}{1801} .
$$

For the simplicity, we did not incorporate time dependence yet, but our analysis extends also to the case of time dependent conductivity parameters. If the analytic solution of the governing equation (32) is

$$
\eta(t, x)=\frac{t^{2}}{4} \cdot\left(1+\left(x-\frac{1801}{1803}\right)^{2}\right)
$$

we can verify that the equalities

$$
\begin{aligned}
-\frac{1}{\kappa_{\mathrm{eff}}(t, 0)} I(t) & =\partial_{x} \eta(t, 0)=\frac{t^{2}}{2} \frac{1801}{1803} \\
\frac{1}{\sigma_{\mathrm{eff}}(t, 1)} I(t) & =\partial_{x} \eta(t, 1)=-\frac{t^{2}}{2}\left(1-\frac{1801}{1803}\right)
\end{aligned}
$$

hold true such that $\partial_{x} \eta(t, 0)$ and $\partial_{x} \eta(t, 1)$ corresponds to $u_{l}$ and $u_{r}$ in $(1)$, where $I(t)=10^{-3} \cdot \frac{1801}{1803} t^{2}$. These show that the boundary conditions in (27) are satisfied.

Using all parameters we can give $C_{\mathrm{dl}}(x)$ such that $\eta$ in (39) is the solution of (32) with the boundary conditions in (27).

It is justified to use the numerical method in Section 3 to approximate $u$ since the Assumptions 1, 2 and 3 are satisfied:

- According to (37) and the choice of the linear kinetics,

$$
\partial_{3} F(t, x, u)=c(x) \alpha \frac{F}{R T},
$$

which is bounded.

- The coefficient functions $p$ and $q$ given in (37) are obviously positive.

- The inequalities in Assumption 3 have been verified consecutively in the time steps during the simulations. These results are shown in Figure 1. One can see that using a reasonably accurate space discretization we can simulate underlying process over sufficiently long time. 


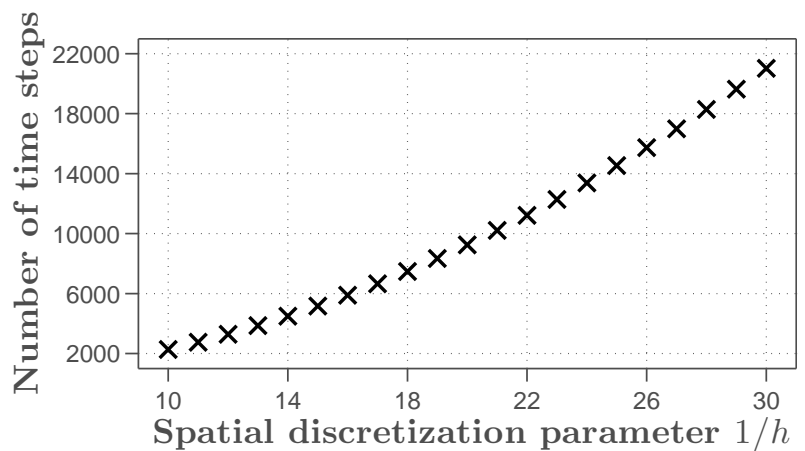

Figure 1: Number of steps $N$ with step length $\tau=1 \mathrm{~s}$ until Assumption 3 is satisfied vs. the number $n$ of the grid points on the interval $I=1 \mathrm{~cm}$.

\subsection{Numerical results}

We present some numerical results here corresponding to the model problem discussed in Section 4.3. The analytic and the numerical solution are compared at $T=1$ in Fig. 2 for a single parameter set.

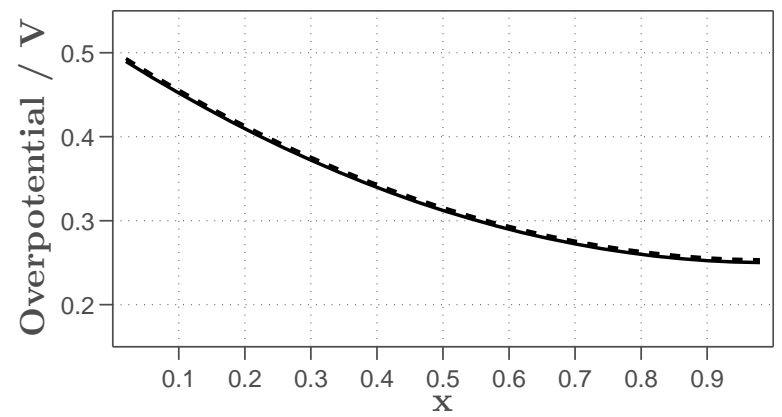

Figure 2: Analytic solution (39) of (32) (continuous line) and the numerical approximation (dashed line) using the method in (2) with $T=1, N=25$ and $\tau=0.01$ for the test problem in Section 4.3. The remaining parameters are given in the Appendix.

We investigated the order of convergence in the $\|\cdot\|_{\infty}$ norm experimentally with respect to the spatial discretization. For this we have consecutively refined the grid and the time step simultaneously such that the ratio $\frac{\tau}{h^{2}}$ is kept at constant level. Accordingly, in the figures we only investigate the dependence of the $\|\cdot\|_{\infty}$-norm error on the number $\frac{1}{h}$ of the spatial grid points. The corresponding results are shown in Figure The numerical results confirm our expectation in Section 3: we can fit accurately a line of slope -2 to the log-log data, which shows a second order convergence with respect to the spatial discretization parameter, see Fig. 3. 

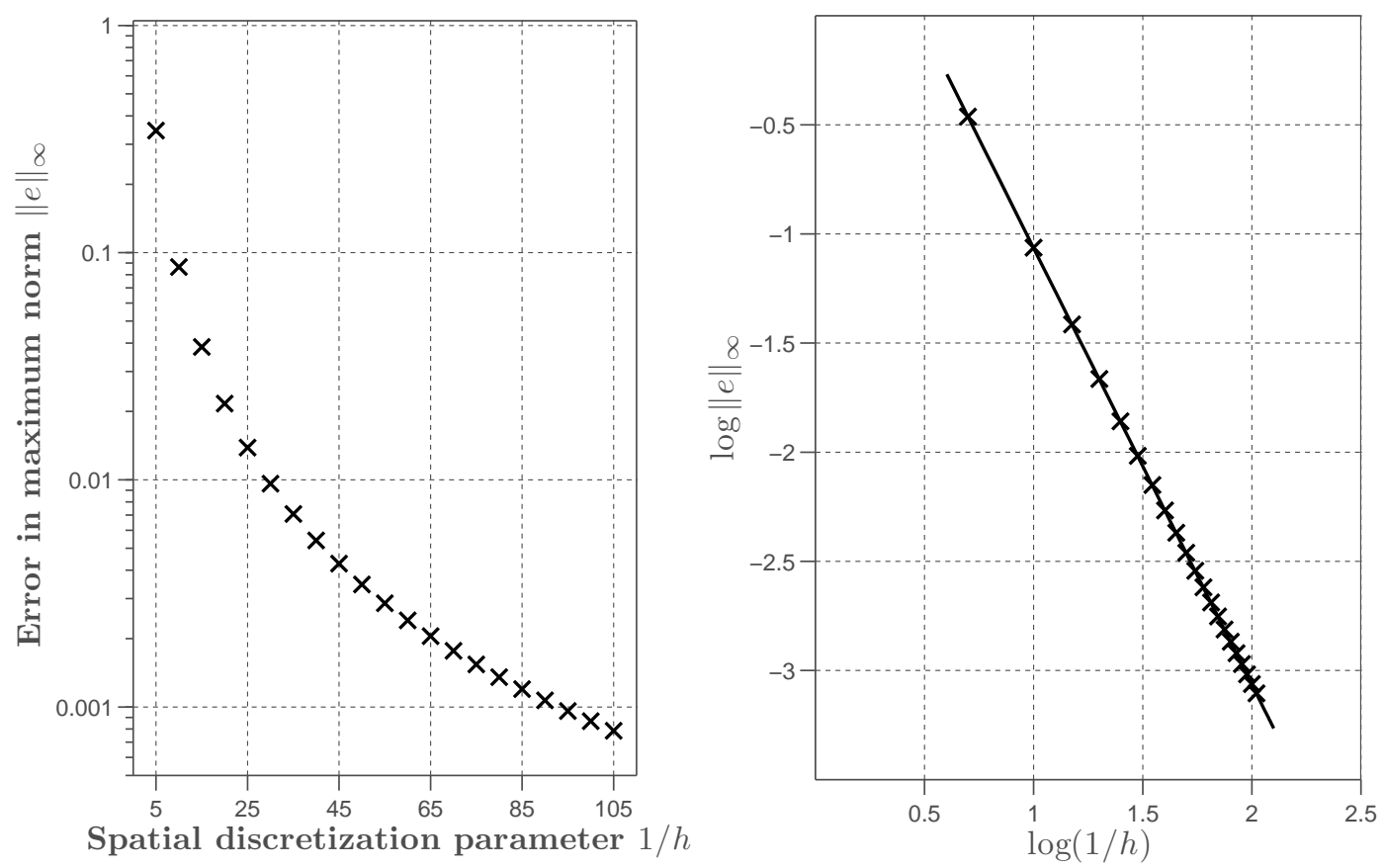

Figure 3: $\|\cdot\|_{\infty}$ norm error in the numerical solution for the test problem in Section 4.3 vs. the spatial discretization parameter (left side). Log-log plot of the error vs. the spatial discretization parameter and a fitted line with slope -2 (left side). 


\section{Appendix}

\begin{tabular}{lrr} 
Symbol & Description & Unit \\
\hline$a$ & Specific interfacial area & $\mathrm{cm}^{-1}$ \\
$C_{\mathrm{dl}}$ & Double-layer capacitance & $\mathrm{F} / \mathrm{cm}^{2}$ \\
$E_{\text {cell }}$ & Cell potential & $\mathrm{V}$ \\
$E_{\mathrm{OC}}$ & Open circuit potential & $\mathrm{V}$ \\
$F$ & Faraday constant $(96487)$ & $\mathrm{C} / \mathrm{mol}^{2}$ \\
$I$ & Total cell current density & $\mathrm{A} / \mathrm{cm}^{2}$ \\
$i_{0}$ & Exchange current density at the cathode & $\mathrm{A} / \mathrm{cm}^{2}$ \\
$i_{0}^{a}$ & Exchange current density at the anode & $\mathrm{A} / \mathrm{cm}^{2}$ \\
$i_{1}$ & Solid phase current density at the cathode & $\mathrm{A} / \mathrm{cm}^{2}$ \\
$i_{2}$ & Solution phase current density at the cathode & $\mathrm{A} / \mathrm{cm}^{2}$ \\
$i_{f}$ & Faradaic current density & $\mathrm{A} / \mathrm{cm}^{3}$ \\
$j_{D}$ & Limiting current at the cathode & $\mathrm{A} / \mathrm{cm}^{2}$ \\
$L$ & Thickness of the cathode & $\mathrm{cm}$ \\
$R$ & Universal gas constant (8.3144) & $\mathrm{J} / \mathrm{molK}^{2}$ \\
$T$ & Cell temperature & $\mathrm{K}$ \\
$V^{*}$ & Potential loss at the cathode & $\mathrm{V}$ \\
$W_{\mathrm{mem}}$ & Membrane thickness & $\mathrm{cm}$ \\
$\alpha$ & Transfer coefficient in the cathode & \\
$\alpha_{a}^{a}$ & Anodic transfer coefficient at the anode & \\
$\alpha_{c}^{a}$ & Cathodic transfer coefficient at the anode & \\
$\eta$ & Overpotential at the cathode & $\mathrm{V}$ \\
$\eta^{a}$ & Overpotential at the anode & $\mathrm{V}$ \\
$\nu^{2}$ & Solid phase potential & $\mathrm{V}$ \\
$\phi_{1}$ & Solution phase potential & $\mathrm{V}$ \\
$\phi_{2}$ & Dimensionless Exchange curent density & $\mathrm{S} / \mathrm{cm}$ \\
$\kappa_{\mathrm{eff}}$ & $\mathrm{S} / \mathrm{cm}$ \\
$\sigma_{\mathrm{eff}}$ & $\mathrm{S} / \mathrm{cm}$ \\
$\sigma_{\mathrm{m} \text { mem }}$ & Effective solution phase conductivity \\
\hline & Effective solid phase conductivity &
\end{tabular}

\section{References}

[1] U. M. Ascher, S. J. Ruuth, and R. J. Spiteri. Implicit-explicit Runge-Kutta methods for timedependent partial differential equations. Appl. Numer. Math., 25(2-3):151-167, 1997. Special issue on time integration (Amsterdam, 1996).

[2] U. M. Ascher, S. J. Ruuth, and B. T. R. Wetton. Implicit-explicit methods for time-dependent partial differential equations. SIAM J. Numer. Anal., 32(3):797-823, 1995.

[3] K. Burrage, W. H. Hundsdorfer, and J. G. Verwer. A study of $B$-convergence of Runge-Kutta methods. Computing, 36(1-2):17-34, 1986.

[4] J. Frank, W. Hundsdorfer, and J. G. Verwer. On the stability of implicit-explicit linear multistep methods. Appl. Numer. Math., 25(2-3):193-205, 1997. Special issue on time integration (Amsterdam, 1996). 
[5] D. Hoff. Stability and convergence of finite difference methods for systems of nonlinear reactiondiffusion equations. SIAM J. Numer. Anal., 15(6):1161-1177, 1978.

[6] T. Koto. IMEX Runge-Kutta schemes for reaction-diffusion equations. J. Comput. Appl. Math., 215(1):182-195, 2008.

[7] A. Kriston, G. Inzelt, I. Faragó, and T. Szabó. Simulation of the transient behavior of fuel cells by using operator splitting techniques for real-time applications. Computers and Chemical Engineering.

[8] S. Litster and N. Djilali. Mathematical modelling of ambient air-breathing fuel cells for portable devices. Electrochimica Acta, 52(11):3849-3862, 2007.

[9] J. Newman and K. E. Thomas-Alyea. Electrochemical systems. John Wiley \& Sons, Inc., Hoboken, New Yersey, third edition, 2004.

[10] M. Robinson. IMEX method convergence for a parabolic equation. J. Differential Equations, 241(2):225-236, 2007.

[11] V. R. Subramanian, V. Boovaragavan, and V. D. Diwakar. Toward real-time simulation of physics based lithium-ion battery models. Electrochemical and Solid-State Letters, 10(11):A255-A260, 2007.

[12] J. G. Verwer. Convergence and order reduction of diagonally implicit Runge-Kutta schemes in the method of lines. In Numerical analysis (Dundee, 1985), volume 140 of Pitman Res. Notes Math. Ser., pages 220-237. Longman Sci. Tech., Harlow, 1986.

[13] C. Ziegler, H. M. Yu, and J. O. Schumacher. Two-phase dynamic modeling of pemfcs and simulation of cyclo-voltammograms. Journal of The Electrochemical Society, 152(8):A1555-A1567, 2005. 